\title{
A CONTRIBUIÇÃO DA MEDICINA BASEADA EM EVIDÊNCIAS PARA A INTRODUÇÃO DE NOVO CONHECIMENTO NA PRÁTICA CLÍNICA
}

\author{
Fauze MALUF-FILHO ${ }^{1}$
}

\begin{abstract}
RESUMO - Com a vasta produção científica e o surgimento de novas terapias e equipamentos, torna-se fundamental que o gastroenterologista lance mão de instrumentos que o auxiliem na avaliação crítica do "novo" conhecimento, que poderá ou não ser incorporado a sua prática clínica. A medicina baseada em evidências consiste neste instrumento. Associando conceitos da informática médica e da epidemiologia clínica, a medicina baseada em evidências tem como único e maior objetivo o paciente. Assim, na análise crítica do "novo conhecimento", o movimento da medicina baseada em evidências valoriza os ensaios clínicos corretamente aleatorizados, com grupo controle e casuística adequados, com desfechos claramente expostos e clinicamente válidos. Quando os resultados dos ensaios clínicos são conflitantes, o movimento valoriza o instrumento da revisão sistemática, de preferência tratada do ponto de vista estatístico, conhecida como metanálise. Utilizando estas ferramentas, torna-se mais objetiva e ética a incorporação de novos tratamentos à prática gastroenterológica.

DESCRITORES - Medicina baseada em evidências. Educação de pós-graduação em medicina.
\end{abstract}

O processo de tomada de decisões médicas envolve a utilização de conhecimentos científicos de veracidade facilmente verificável, bem como de conhecimentos, experiências e habilidades de valor científico menos reconhecido. Estes últimos constituem-se fundamentalmente de conceitos transmitidos, em geral oralmente, por professores, preceptores ou algum " $R$ " (residente) mais antigo. O mais notável é que dificilmente o estudante ou residente que recebe estes conceitos reflete sobre os mesmos, procura por atualizações sobre o tema ou sequer verifica a origem destes conhecimentos. Recentemente, este modelo de ensino, onde informações estáticas são oferecidas cumulativamente para estudantes "passivos", foi desafiado pelo paradigma proposto pela Medicina Baseada em Evidências (MBE) ou "Evidence-Based Medicine", em $1992^{(2)}$. Na definição de seus criadores, trata-se do uso da melhor evidência científica para a tomada de decisões para determinado paciente ${ }^{(3)}$. A principal crítica a tal movimento e, principalmente a esta definição é: "Não é o que costumamos fazer em nossa prática diária?" Claramente a reposta é Não. No modelo proposto pela MBE, frente a qualquer indagação sobre diagnóstico, prognóstico e tratamento, o profissional de saúde acessa determinado banco de dados (como o Pubmed ${ }^{\circledR}$, por exemplo), inserindo algumas palavras-chaves, recupera os artigos relevantes, qualifica a evidência contida nas publicações (avaliando a qualidade dos artigos) e aplicando as informações obtidas para o contexto clínico em questão (isto é, para aquele doente específico), toma decisões que são quantificáveis através de razões probabilísticas e de medidas de associação epidemiológica. O grupo criador deste modelo exemplifica-o com o caso clínico de um homem de 48 anos que se apresenta à emergência com $1^{\circ}$ episódio de convulsão do tipo grande mal, sem história de trauma ou ingestão alcoólica, com estudo tomográfico de crânio normal e eletroencefalograma sem alterações conclusivas. No momento da alta, o paciente indaga sobre o risco de novas crises convulsivas (prognóstico) e se alguma medicação deve ser prescrita para evitálas. No modelo antigo, o médico residente se dirigirá ao preceptor e este, ao assistente, que o informará de que há alta chance de nova crise (quanto exatamente?) e que o paciente deverá tomar anticonvulsivante por tempo indefinido. No modelo atual, o médico residente procura um terminal conectado à Internet e faz busca no Pubmed ${ }^{\circledR}$, avaliando os artigos relevantes para o seu paciente. Após cerca de 30 a 60 min, pode informá-lo de que o risco de recurrência é de 40 a $50 \%$ no primeiro

Disciplina do Programa de Pós-graduação em Cirurgia do Aparelho Digestivo da Faculdade de Medicina da Universidade de São Paulo: "Medicina Baseada em Evidências: Fundamentos e Aplicabilidade na Investigação Científica"

Correspondência: Prof. Fauze Maluf-Filho - Rua Olegário Mariano, 488 - 05612-000 - São Paulo, SP. 
ano, caindo esta cifra para $20 \%$ passados 18 meses da primeira crise. O paciente recebe a prescrição de anticonvulsivante por 18 meses e recomendação de retornar regularmente ao médico assistente, para possível interrupção do medicamento passado este período, se assintomático.

Estes mesmos princípios devem reger a introdução de novo conhecimento na prática clínica. Quando se fala sobre tratamento, a melhor evidência ou aquela mais confiável é a que tem origem de estudos controlados com alocação aleatória, ou os conhecidos "RCTs - randomized controlled trials". Neles, o novo tratamento (experimento) é comparado com a terapêutica considerada padrão-ouro para o manejo da doença ou com o placebo, se este tratamento não existir. A alocação aleatória ou "randomização" (anglicismo da palavra inglesa "random" - ao acaso) garante que os dois grupos a serem comparados sejam semelhantes quanto aos dados demográficos (sexo, idade, cor), gravidade da enfermidade e frequência de outras comorbidades. Evita-se, assim, o viés de seleção, onde pacientes mais graves se acumulam em determinado grupo de tratamento. Evitando-se este viés, se houver diferença entre os grupos, no que se refere aos resultados, poder-se-á inferir que esta diferença foi fruto dos tratamentos e não das condições prévias dos pacientes. Fundamental também é definir qual desfecho clínico será medido e como fazê-lo. São comuns estudos demonstrarem que determinado tratamento provoca menor frequência de um desfecho secundário, sem informar sobre o evento principal. Num estudo comparando o uso de lidocaína versus placebo em pacientes infartados, qual será o desfecho mais importante a ser mensurado: frequência de arritmias ventriculares ou mortalidade? Certamente, o segundo. De fato, mesmo na era pós-trombólise para o tratamento do infarto agudo do miocárdio, vários estudos sugerem que o uso de lidocaína não está indicado, pois, embora reduza a frequência de arritmias ventriculares, aumenta a mortalidade entre os pacientes ${ }^{(4)}$. Ainda, ao se analisar qualquer estudo que sugere a introdução de nova droga ou operação, devese avaliar se o número de pacientes incluídos é suficiente para demonstrar a diferença que se quis provar entre os tratamentos. Exemplo clássico deste problema são os estudos que lidam com tratamento de hemorragia digestiva provocada por úlcera péptica. Uma vez que a mortalidade gerada por esta situação é de 5\%-8\%, ao se comparar dois grupos de 100 pacientes submetidos a dois tipos diferentes de tratamento para a hemostasia daquela úlcera, espera-se que ocorram cerca de cinco a oito óbitos em cada grupo. Assim, a maioria dos trabalhos sobre este tema consegue demonstrar a superioridade de um tratamento sobre o outro em relação às taxas de ressangramento, volume de hemotransfusão, permanência hospitalar, mas não conseguem demonstrar impacto sobre a mortalidade. Fica-se sem saber se esta redução estatística da mortalidade não ocorreu porque, de fato, os tratamentos têm resultados semelhantes neste aspecto, ou se não foi estudado número suficiente de pacientes para se demonstrar a diferença. Esta última situação configura que o estudo não teve poder estatístico ("unpowered trial") para provar a hipótese (ou diferença entre os tratamentos), também conhecido como erro tipo II que não deixa de ser um tipo de falso-negativo. O contrário raramente acontece, isto é, o estudo demonstrou que houve diferença entre os tratamentos, mas em realidade, esta diferença ocorreu ao acaso, isto é, foi um falso-positivo, pois se fixa o valor "estatisticamente significante" em inferior a 5\% ou o conhecido $P<0,05$. Significa que a chance de determinada diferença ter ocorrido ao acaso é inferior a $5 \%$ e é de $95 \%$ de que a diferença ocorreu pela superioridade de um tratamento em relação ao outro. Outra impropriedade frequentemente detectada nos estudos é a maneira de coletar os resultados. Idealmente, quem coleta os dados e o paciente incluído no protocolo não devem saber a que grupo este pertence, o que transforma o estudo em "duplo-cego". Se o próprio médico intensivista sabe qual tratamento o paciente está recebendo afere a medida do desfecho do estudo, é possível que ocorram viéses ("bias" da literatura inglesa) no momento da aferição dos dados. Ainda, a análise dos resultados deve ser por intenção de tratamento ("ITT - intention to treat") e não por protocolo ("PP - per protocol"). Por exemplo, um determinado paciente, alocado para o grupo experimento, não consegue ser submetido a este tratamento porque, por exemplo, desenvolve uma alergia à droga. Consiste em erro metodológico grosseiro simplesmente colocar este paciente no grupo placebo (já que ele não conseguiu receber todas as doses da droga "ativa") e analisá-lo como tal. Esta é análise PP. $\mathrm{O}$ paciente deve ser analisado como se tivesse recebido todo o tratamento ("ITT"), porque afinal, esta é a vida real e o estudo deve aferir a efetividade daquele tratamento. Em resumo, são as melhores evidências científicas sobre determinado tratamento de uma doença aquelas extraídas de estudos controlados, duplo-cegos, de alocação aleatória, com análise por intenção de tratamento, com poder estatístico suficiente, com desfechos válidos e adequadamente mensurados.

Muitas vezes, encontra-se na literatura análise de vários destes estudos, como se fossem uma única coorte de pacientes. Trata-se da revisão sistemática. Quando os resultados da revisão sistemática são analisados estatisticamente, define-se metanálise. Vários autores consideram a metanálise evidência ainda mais poderosa do que um "RCT" bem conduzido. As revisões sistemáticas devem incluir apenas estudos controlados, "randomizados", de alta qualidade, que respeitem os preceitos acima expostos. Conclui-se que mesmo as revisões sistemáticas e metanálises merecem olhar crítico, antes que suas conclusões sejam simplesmente aplicadas na prática clínica. Deve-se ter em mente que não existem "RCT" ou metanálises" sobre todos os assuntos. Assim, muitas vezes, nossas decisões se baseiam em estudos que analisaram retrospectivamente o resultado de determinado experimento (intervenção ou droga) ou ainda, que acompanharam prospectivamente pacientes submetidos àquela intervenção, sem compará-los a grupo controle (desenho de estudo conhecido como ensaio clínico não controlado). O médico opta pelo experimento pois os resultados destes estudos são superiores àqueles obtidos historicamente com outros tratamentos. Ou ainda, porque está diante de condição de reconhecida alta letalidade, em que o estudo demonstrou resultado do tipo "tudo ou nada", isto é, quase todos os pacientes submetidos a intervenção sobreviveram, enquanto o desfecho esperado para todos era desfavorável.

Quando da introdução de novos conhecimentos na prática clínica, deve-se quantificar o impacto deste novo tratamento, 
através das medidas de associação como "odds ratio" ou razão de chances, "relative risk" ou risco relativo, "relative risk reduction" ou redução relativa do risco, "absolute risk reduction" ou redução absoluta do risco e o "number needed to treat" - NNT ou número necessário para tratar. Suponhamos que determinado tratamento reduza a mortalidade de uma doença de $30 \%$ para $10 \%$. A redução absoluta do risco, com este tratamento, é de $20 \%$ (30-10), a redução relativa do risco, de $67 \%(20 / 30)$ e o NNT, de $5(1 \% / 20 \%)$. Esta última medida de associação indica que, ao aplicarmos o novo tratamento em cinco pacientes, evitar-se-á a morte em um. Estas medidas, especialmente o NNT, são fundamentais para a análise da relação custo-benefício de um determinado tratamento. Suponhamos tratamento de alto custo para situação de alta letalidade cujo resultado tenha NNT muito elevado (mais de 100, por exemplo). Dificilmente, este tratamento será aplicado para todos os pacientes portadores daquela condição. Nesta situação, devem-se identificar subgrupos de pacientes que se beneficiem "mais" dos que os outros, reduzindo o NNT. Por outro lado, o tratamento tríplice para $H$. pylori apresenta NNT próximo de 1 , quando o objetivo é a erradicação da bactéria; por outro lado, este número é 20, quando se analisa o alívio da dispepsia .

Estes conceitos se tornam fundamentais nos tempos atuais se o médico quiser analisar criteriosamente se o que há de mais novo ou mais avançado é, de fato, o melhor a seu paciente. Os exemplos se acumulam em nossa área de atuação. No início deste século, foram propostas algumas terapias endoscópicas antirrefluxo. Por mais de 4 anos, tudo o que se apresentou foram ensaios clínicos em que pacientes com graus leves de esofagite, sem hérnia de hiato, de peso normal, foram submetidos a injeções, plicaturas e outras intervenções na transição esôfago-gástrica. Os desfechos clínicos buscados pelos estudos foram nitidamente intermediários: redução no uso do inibidor de bomba de prótons, redução no tempo de exposição ao ácido medido pela pHmetria, redução dos "escores" de sintomas. Naquele momento, os desfechos válidos ensinados na graduação, a saber, controle de sintomas e cicatrização da esofagite, foram esquecidos, ou "afrouxados" para se permitir a realização destes protocolos.

Não se deve esquecer de que, ao final do processo de busca pela melhor evidência sobre o tipo de tratamento a ser ministrado, ou ao introduzir novo conhecimento na prática clínica, o médico deverá individualizar a terapêutica, isto é, avaliar se a evidência obtida se aplica àquele determinado paciente e se algumas adaptações devem ser feitas. $\mathrm{O}$ que o conceito da MBE prevê é o perfeito equilíbrio entre a melhor evidência científica e a experiência ("viés") pessoal, onde o agente de saúde enxerga o enfermo além de sua doença ${ }^{(1)}$.

Maluf-Filho F. The importance of evidence-based medicine concepts for the clinical practitioner. Arq Gastroenterol. 2009;46(2):87-9.

ABSTRACT - The acceptance or refusal of new therapies and technologies pose a formidable problem for the daily practice of the gastroenterologist. It is of utmost importance to adopt clear strategies to select the true evidences from the huge amount of new medical information. Gathering fundamental concepts of clinical epidemiology and medical informatics, evidence-based medicine (EBM) build up those strategies keeping the patient as the main objective of its application. The evidence-based medicine movement values the randomized controlled clinical trials, with adequate sample size and clear and valid outcomes. Facing conflicting results from those trials, evidence-based medicine values the systematic review and whenever possible its statistical counterpart, the meta-analysis. Evidence-based medicine provides valuable tools for the ethic and less subjective incorporation of new treatments and technologies in gastroenterology.

HEADINGS - Evidence-based medicine. Education, medical, graduate.

\section{REFERÊNCIAS}

1. Bernardo WM, Nobre MC. Prática clínica baseada em evidência. Rio de Janeiro: Elsevier; 2006. 238p.

2. Evidence-Based Medicine Working Group. Evidence-based medicine. A new approach to teaching the practice of medicine. JAMA. 1992;268:2420-5.

3. Sackett DL, Straus S, Richardson S, Rosenberg W, Haynes RB. Evidence-based medicine: how to practice and teach EBM. 2nd ed. Londres: Churchill Livingstone 2000 .

4. Sadowski ZP, Alexander JH, Skrabucha B, Dyduszynski A, Kuch L, Nartowicz E, Swiatecka G, Kong DF, Granger CB. Am multicenter randomized trial and a systematic overview of lidocaine in acute myocardial infarction. Am Heart J. 1999;137:792-8.

Recebido em 7/1/2009. Aprovado em 2/3/2009. 